\title{
Exame físico na enfermagem: avaliação do conhecimento teórico-prático
}

\begin{abstract}
RESUMO | Objetivo: Verificar o conhecimento teórico-prático dos enfermeiros sobre a técnica do exame físico céfalo-caudal em um hospital universitário da cidade de São Paulo. Método: Estudo descritivo-exploratório de abordagem quantitativa realizado com 51 enfermeiros de unidades de internação e terapia intensiva adulto, submetidos a um questionário estruturado. A análise dos resultados foi feita por meio da estatística descritiva e análise inferencial por associação entre as variáveis de interesse. Resultados: Os dados revelaram índices de acertos acima de 70\%. A graduação foi considerada o período de maior aquisição de conhecimento sobre o exame físico $(p=0,039)$. O tempo de formação e atuação foram associados a maior escolaridade $(p<0,05)$, a qual foi determinante para prática do exame físico $(73,8 \%)$. Conclusão: os achados desta investigação reforçam a importância da educação permanente junto aos enfermeiros para a prática do exame físico com objetivo de manter uma assistência de qualidade orientada para a segurança do paciente.
\end{abstract}

Palavras-chaves: Exame Físico; Processo de Enfermagem; Educação em Enfermagem.

ABSTRACT | Objective: To verify the theoretical and practical knowledge of nurses on the technique of cephalo-caudal physical examination at a university hospital in the city of São Paulo. Method: Descriptive-exploratory study with a quantitative approach carried out with 51 nurses from inpatient and adult intensive care units, submitted to a structured questionnaire. The analysis of the results was performed using descriptive statistics and inferential analysis using evidence of association between the variables of interest. Results: The data revealed success rates above $70 \%$. Graduation was considered the period of the greatest acquisition of knowledge about the physical examination $(p=0.039)$. The time since graduation was associated with higher education $(p<0.05)$, which was decisive for the practice of physical examination (73.8\%). Conclusion: the findings of this investigation reinforce the importance of continuing education with nurses for the practice of physical examination in order to maintain quality care oriented to patient safety.

Keywords: Physical Examination; Nursing Process; Education, Nursing.

RESUMEN | Objetivo: verificar los conocimientos teóricos y prácticos de las enfermeras sobre la técnica del examen físico cefalo-caudal en un hospital universitario de la ciudad de São Paulo. Método: estudio descriptivo-exploratorio con enfoque cuantitativo realizado con 51 enfermeras de unidades de cuidados intensivos para pacientes hospitalizados y adultos, sometidas a un cuestionario estructurado. El análisis de los resultados se realizó mediante estadística descriptiva y análisis inferencial utilizando evidencia de asociación entre las variables de interés. Resultados: Los datos revelaron tasas de éxito superiores al $70 \%$. La graduación se consideró el período de mayor adquisición de conocimiento sobre el examen físico $(p=0.039)$. El tiempo transcurrido desde la graduación se asoció con la educación superior $(\mathrm{p}<0.05)$, lo cual fue decisivo para la práctica del examen físico (73.8\%). Conclusión: los resultados de esta investigación refuerzan la importancia de la educación continua con enfermeras para la práctica del examen físico a fin de mantener una atención de calidad orientada a la seguridad del paciente.

Palavras claves: Examen Físico; Proceso de Enfermería; Educación en Enfermería.

\section{Thais de Lima}

Graduanda em Enfermagem pela Universidade Federal de São Paulo. São Paulo (SP), Brasil.

\section{Carla Roberta Monteiro}

Enfermeira. Doutora em ciências. Professora Adjunta da Escola Paulista de Enfermagem da Universidade Federal de São Paulo. São Paulo (SP), Brasil.

\section{Tânia Arena Moreira Domingues}

Enfermeira. Doutora em Enfermagem na Saúde do Adulto. Professora associada da Escola Paulista de Enfermagem do Departamento de Enfermagem Clínica e Cirúrgica, Disciplina Enfermagem Fundamental, da Universidade Federal de São Paulo. São Paulo (SP), Brasil.

Recebido em: 06/02/2020

Aprovado em: 29/03/2020

\section{Ana Paula Dias de Oliveira}

Enfermeira. Mestre em Enfermagem. Técnica Administrativa no Ensino do Departamento de Enfermagem Clínica e Cirúrgica da Escola Paulista de Enfermagem da Universidade Federal de São Paulo. São Paulo (SP), Brasil.

\section{Cassiane Dezoti da Fonseca}

Enfermeira. Doutora em Ciências da Saúde. Professora Adjunta do Departamento de Enfermagem Clínica e Cirúrgica da Escola Paulista de Enfermagem da Universidade Federal de São Paulo. São Paulo (SP), Brasil.
INTRODUÇÃO

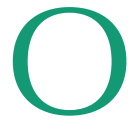
exame físico é uma habilidade inerente a prática assistencial de diversos profissionais da área da saúde. No entanto, com o surgimento de novas tecnologias diagnósticas sua aplicação beira-leito tem se tornado cada vez mais incipiente. Há cerca de dois milênios Hipócrates já enfatizava a percepção clínica pela "visão, toque, audição, olfato, paladar e compreensão", configurando-se nos principais pilares da prática do exame físico ${ }^{1,2}$.

A interpretação de achados e a identificação das anormalidades são habilidades adquiridas no decorrer do desenvolvimento profissional e são fundamentadas pelos conhecimentos dos aspectos anato- 
mofisiológicos e fisiopatológicos ${ }^{3,4}$. Neste sentido, a prática clínica é organizada por meio de um bom raciocínio clínico aliado ao pensamento crítico ${ }^{5}$.

A enfermagem é a profissão da área da saúde que mais atua beira-leito e suas atividades, a saber o exame físico, são sustentadas por um método de trabalho organizado, o Processo de Enfermagem (PE), que utiliza como referencial a Teoria das Necessidades Humanas Básicas de Wanda Aguiar Horta. Essa teoria classifica as necessidades humanas em psicobiológicas, psicossociais e psicoespirituais ${ }^{6,7}$. A regulamentação da resolução 358/2009 pelo COFEN tornou obrigatório no Brasil a implementação da Sistematização da Assistência de Enfermagem (SAE) e do Processo de Enfermagem em todos os cenários onde se dá o exercício profissional do enfermeiro 8. Este método de trabalho deve ser realizado de forma deliberada e inter-relacionada por meio de cinco etapas: coleta de dados, diagnóstico, prescrição, intervenção e evolução de enfermagem. O exame físico está inserido nessas etapas e atende às necessidades psicobiológicas orientadas por Horta ${ }^{5,6,8}$.

Diversos estudos têm vislumbrado a temática do exame físico e as lacunas associadas entre o conhecimento e a prática. Neles foram observados aspectos que envolvem dissociação teórico-prática, desinteresse por parte dos enfermeiros em rever conteúdos de anamnese e exame físico9, despreparo dos docentes10 e precarização do trabalho ${ }^{4,11}$.

Neste contexto, será que o conhecimento teórico-prático estaria relacionado com a prática do exame físico entre os enfermeiros da área hospitalar? Dessa forma, o presente estudo visa verificar o conhecimento teórico-prático dos enfermeiros sobre a técnica do exame físico céfalo-caudal em um hospital universitário da cidade de São Paulo. Os achados desta investigação podem contribuir para a elaboração de intervenções educativas com o objetivo de aprimoramento deste profissional nesta atividade importante que compõe o método de trabalho da enfermagem.

\section{“}

A enfermagem é a profissão da área da saúde que mais atua beira-leito e suas atividades, a saber o exame físico, são sustentadas por um método de trabalho organizado, o Processo de Enfermagem (PE), que utiliza como referencial a Teoria das Necessidades Humanas Básicas de Wanda Aguiar Horta. Essa teoria classifica as necessidades humanas em psicobiológicas, psicossociais e psicoespirituais ${ }^{6,7}$.
MÉTODO

Trata-se de um estudo descritivo-exploratório, de coorte transversal de abordagem quantitativa, envolvendo uma população de 192 enfermeiros que trabalha em unidades de internação e unidades de terapia intensiva adulto de um hospital universitário da cidade de São Paulo. A amostra foi calculada baseada na fórmula estatística para populações finitas, resultando em 51 enfermeiros, com um nível de confiança de $90 \%$ e erro amostral de $8 \%$. A seleção deu-se de forma aleatória simples, tendo sido após envio do convite para participação da pesquisa e do questionário estruturado por meio de rede social ou mala direta eletrônica, adotando os seguintes critérios de inclusão: enfermeiros formados e atuantes nas unidades de internação e unidades de terapia intensiva adulto e os critérios de exclusão: enfermeiros da pediatria, visto que há inúmeras peculiaridades que diferenciam o cuidado do paciente adulto com o pediátrico.

Os dados foram coletados no período compreendido de fevereiro a maio de 2019 por meio de um formulário eletrônico que abordava características demográficas, histórico e atuação profissional, questões sobre a satisfação da prática do exame físico e questionário de avaliação do conhecimento.

O questionário de avaliação do conhecimento teórico-prático do exame físico foi elaborado pela pesquisadora. Posteriormente foi avaliado e corrigido por experts que atuam em disciplinas de semiologia e semiotécnica de universidades federais brasileiras.

Os dados foram tabulados em planilha eletrônica Excel e processados no software Statistical Package for the Social Sciences (SPSS), versão 22. A análise dos resultados foi feita por meio da estatística descritiva e análise inferencial por provas de associação entre as variáveis de interesse. A análise de associação entre variáveis de interesse foi feita com o Teste exato de Fisher, Mann Whitney e Kruskal Wallis. Em todas as análises realizadas foi utilizado o nível de significância de 5\%. 
Dificuldades para realizar o exame físico

\begin{tabular}{l} 
Falta de tempo \\
Recursos humanos deficiente \\
\hline Falta de instrumentos \\
\hline Falta de privacidade \\
Não enfrento dificuldade para o exame físico \\
Duas dificuldades \\
Mais de duas dificuldades
\end{tabular}

\section{Segurança na realização do exame físico}

Sim
Não
Fonte: Dados da pesquisa, 2019. *Teste exato de Fisher.

$\begin{array}{cc}9 & 24,3 \\ 0 & 0 \\ 2 & 5,4 \\ 1 & 2,7 \\ 9 & 24,3 \\ 10 & 27 \\ 6 & 16,3\end{array}$

$\begin{array}{cccc}31 & 83,8 & 12 & 85,7 \\ 6 & 16,2 & 2 & 14,3\end{array}$

1

A tabela 3 revela a comparação do tempo de formado e tempo de atuação com as diversas variáveis do estudo. Observou-se que o tempo de formado e o tempo de atuação apresentaram diferen- ças significativas nas variáveis gênero e escolaridade. Destaca-se na escolaridade que quanto maior o tempo, maior o aperfeiçoamento profissional.

Na tabela 4 observa-se a escolha das técnicas propedêuticas pelos participantes nos diversos sistemas corporais. A inspeção foi a técnica propedêutica com maior aplicação e esteve presente em todos os sistemas. A palpação também foi

\section{Tabela 3. Relação do tempo de formado e de atuação com diversas variáveis do estudo. São Paulo, 2019.}

\section{Tempo de Formado (meses)}

Variáveis

Gênero

Masculino

Feminino

Escolaridade

Ensino Superior

Especialização

Mestrado

Local de trabalho

Unidade de internação

Unidade de terapia intensiva

Período de trabalho

Matutino

Vespertino

Noturno

Dois períodos

\section{Segurança na realização}

do exame físico

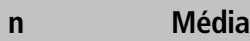

Média

4

47

20,2

79,3

$\begin{array}{cc}9 & 27,0 \\ 35 & 79,9 \\ 7 & 109\end{array}$

7

37

14

80,3

27,0

79,9

109,7

59,6

16

18

8

11

$\begin{array}{cc}87,5 \\ 11 & 126,0\end{array}$

84,3
49,6
97,5
126,0

$\begin{array}{lll}43 & 71,8 & 51,6 \\ 8 & 90,1 & 86,0\end{array}$

0,835

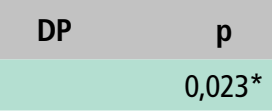

Tempo de atuação (meses)

Média

26,7
57,3

10,0

68,6

$0,003 \&$

23,9

59,0

46,1

$$
0,427
$$

62,7

39,1

0,118

50,1
56,3
63,3
59,3

69,8
48,8

7,2

7,2

71,3

100,5

0,547

$<0,001 \&$

Nimo

Fonte: Dados da pesquisa, 2019. * Teste de Mann Whitney; \& Teste de Kruskal Wallis. 
descrita em todos os sistemas corporais, no entanto, apresentou índices menores nos genitais e sistema circulatório. Com relação a ausculta, destacam-se aplicações nos sistemas circulatório, respiratório e digestório. Por outro lado, curiosa- mente, os sistemas neurológico/Cabeça e pescoço $(5,8 \%)$ e urinário $(3,9)$ apresentaram aplicabilidade para essa técnica propedêutica. A percussão obteve maiores índices de aplicação no sistema digestório (94,1\%), seguidos do respiratório $(82,3 \%)$ e urinário (25,4\%). Apesar de valores discretos, os sistemas Neurológico/Cabeça e Pescoço (1,9\%), genitais (1,9\%), locomotor $(11,7 \%)$ e pele e anexos $(1,9 \%)$ apresentaram aplicabilidade para a técnica propedêutica da percussão.

Tabela 4. Avaliação dos participantes do estudo sobre a utilização das técnicas propedêuticas nos diversos sistemas corporais. São Paulo, 2019.

\section{Técnicas propedêuticas}

\section{Sistemas}

\begin{tabular}{llccc} 
Neurológico/Cabeça e Pescoço & $47(92,1)$ & $3(5,8)$ & $1(1,9)$ & $38(74,5)$ \\
\hline Circulatório & $48(94,1)$ & $42(82,3)$ & $7(13,7)$ & $36(70,5)$ \\
\hline Respiratório & $46(90,1)$ & $44(86,2)$ & $42(82,3)$ & $24(47,0)$ \\
\hline Digestório & $49(96,0)$ & $40(78,4)$ & $48(94,1)$ & $47(92,1)$ \\
\hline Urinário & $45(88,2)$ & $2(3,9)$ & $13(25,4)$ & $37(72,5)$ \\
\hline Genitais & $51(100,0)$ & $0(0,0)$ & $1(1,9)$ & $23(45,0)$ \\
\hline Locomotor & $49(96,0)$ & $0(0,0)$ & $6(11,7)$ & $30(58,8)$ \\
\hline Pele e Anexos & $51(100,0)$ & $0(0,0)$ & $1(1,9)$ & $36(70,5)$ \\
\hline Total & $51(100,0)$ & $51(100,0)$ & $51(100,0)$ & $51(100,0)$
\end{tabular}

\section{DISCUSSÃO}

O conteúdo teórico-prático abordado nesta investigação teve como objetivo avaliar o conhecimento de enfermeiros de unidades de internação e unidades de terapia intensiva para a técnica do exame físico céfalo-caudal. Os dados mostraram maiores índices de acertos do que erros, os quais foram associados à maior frequência da realização da técnica do exame físico. Destaca-se a graduação como o momento mais importante na aquisição do conhecimento do exame físico. O tempo de formado e de atuação foram relacionados com o aperfeiçoamento profissional e gênero. A inspeção e a palpação foram as técnicas propedêuticas com o maior número de utilização no exame físico.

O exame físico constitui a primeira fase do processo de enfermagem e requer a elaboração consistente de um raciocínio clínico. Com isso, o profissional identifica as necessidades do paciente e oferece um plano de cuidados baseado nas respostas humanas com o objetivo de selecionar as intervenções apropriadas e avaliar o resultado alcançado ${ }^{12,13}$.

Neste estudo, os profissionais avaliados demonstraram domínio e assertividade frente às questões sobre o conhecimento teórico-prático do exame físico céfalo-caudal. Esses resultados podem inferir a uma assistência de qualidade com impacto na segurança do paciente ${ }^{14}$.

Trindade et al demonstraram por meio de um estudo exploratório de abordagem qualitativa que enfermeiros compreendem o processo de enfermagem como um método científico e estratégico para qualificação da prática assistencial e reconhecem que sua utilização contribui para a autonomia e valorização da profissão ${ }^{15}$.

O estudo apresentado revelou que um menor número de participantes obteve o conteúdo de exame físico na instituição em que trabalha quan- do comparados aos que obtiveram o conteúdo durante a graduação. Neste contexto, a técnica do exame físico é muito valorizada na graduação, mas é descontinuada principalmente na instituição de trabalho.

O profissional enfermeiro, sem um suporte educacional de longo prazo, tende a não se atualizar autonomamente, isto fica evidenciado por estudos desenvolvidos no Brasil e em demais nacionalidades ${ }^{16,17}$.

Douglas et al avaliaram em 208 estudantes de enfermagem a aplicação das habilidades do exame físico na prática clínica durante a graduação. Constatou-se que das 126 habilidades ensinadas, apenas 5 eram utilizadas com maior frequência, e que algumas habilidades essenciais que deveriam ser realizadas rotineiramente em todos os pacientes foram práticas realizadas raramente ${ }^{18}$. Neste sentido, outra investigação, revelou que de trinta técnicas de avaliação física na enfermagem, vinte e oito eram realizadas ocasionalmente ou raramente ${ }^{19}$. 
Estudo recente que desenvolveu e testou uma escala de avaliação das atitudes e práticas de enfermeiros para o exame físico contribuiu para preencher as lacunas entre o que é ensinado na graduação e a prática. Os resultados revelaram que a execução de um bom exame físico é essencial para o diagnóstico clínico, diminuindo os custos com exames laboratoriais e diagnósticos de imagem, além de fortalecer a relação enfermeiro-paciente ${ }^{20}$.

Neste contexto, a instauração de medidas de educação permanente pode impactar positivamente no nível de conhecimento, na prática e na gestão do cuidado. Uma prática pedagógica motivadora e interativa pode ser utilizada no treinamento e atualização de profissionais, podendo ser um excelente meio de motiva-los ao aprimoramento. Para isso, recomenda-se o uso de metodologias interativas educacionais que se identifiquem com o conceito contemporâneo de ensino, que considera o aprendiz como o centro do processo e construtor da sua aprendizagem.

Por outro lado, esta investigação demonstrou que quanto maior o aperfeiçoamento profissional (especialização e mestrado), maior o tempo de atuação e de formação com associação ao maior número de acertos $(73,8 \%)$. Esses dados reforçam a importância da continuidade dos estudos após a graduação. Silber et al demonstraram que hospitais com melhores ambientes de enfermagem e níveis de pessoal acima da média foram associados a menor mortalidade e cuidado de qualidade em comparação com hospitais sem reconhecimento do ambiente de enfermagem e com pessoal abaixo da média, especialmente para pacientes de maior risco ${ }^{21}$

Os resultados do presente estudo também revelaram precarização do trabalho associado a falta de tempo, instrumentos e recursos humanos escassos. Esse aspecto já havia sido
Estudo recente que desenvolveu e testou uma escala de avaliação das atitudes e práticas de enfermeiros para o exame físico contribuiu para preencher as lacunas entre o que é ensinado na graduação e a prática. Os resultados revelaram que a execução de um bom exame físico é essencial para o diagnóstico clínico, diminuindo os custos com exames laboratoriais e diagnósticos de imagem, além de fortalecer a relação enfermeiropaciente $^{20}$. observado em outros estudos que os relacionou com o comprometimento da técnica ${ }^{4,11}$.

As técnicas propedêuticas avaliadas neste estudo confirmaram a inspeção e a palpação como as técnicas mais utilizadas, semelhantes a estudos que demonstraram a inspeção e a observação geral como as etapas propedêuticas mais utilizadas pelos enfermeiros ${ }^{11,22}$.

Sumariamente, os achados desta investigação reforçam a importância da educação permanente junto aos enfermeiros para a prática do exame físico com objetivo de manter uma assistência de qualidade orientada para a segurança do paciente.

\section{CONCLUSÃO}

Este estudo concluiu que os enfermeiros de unidades de internação e unidade de terapia intensiva adulto apresentaram médias de acerto acima de $70 \%$ com relação às técnicas do exame físico céfalo-caudal. A graduação foi considerada o período de maior aquisição de conhecimento sobre o exame físico. O aperfeiçoamento profissional foi determinante para a melhor prática do exame físico.

Esses dados podem contribuir para a reflexão da prática do exame físico na enfermagem, fortalecendo a qualidade da assistência e destacando o enfermeiro como profissional que possui maior proximidade e empatia com o paciente, tendo importante papel no levantamento de necessidades do paciente, família e /ou comunidade. Assim como, estimular pesquisas de intervenção para aprimoramento no conhecimento da técnica do exame físico na enfermagem.

\section{LIMITAÇõES}

Este estudo foi realizado em um único hospital, o qual apresenta características específicas para 
o ensino. Portanto, novos estudos devem ser desenvolvidos em outros centros para avaliar a diversidade da amostra.
AGÊNCIA FINANCIADORA

Conselho Nacional de Desenvolvimento Científico e Tecnológico- CNPq.

\section{AGRADECIMENTOS}

À Cintia Yurie Yamachi pela contribuição na análise estatística deste trabalho.

\section{Referências}

1. Narula J, Chandrashekhar Y, Braunwald E. Time to Add a Fifth Pillar to Bedside Physical Examination: Inspection, Palpation, Percussion, Auscultation, and Insonation. JAMA Cardiol. 2018[Citado em 2020 Fev. 23];3(4):346-350. Disponível em: sci-hub.tw/10.1001/jamacardio.2018.0001.

2. Elnicki DM. Bayes' theorem and the physical examination: probability assessment and diagnostic decision making. Acad Med. 2011 [Citado em 2020 Fev. 23];86(5):618-627. Disponível em: https://www.ncbi.nlm.nih.gov/pmc/ articles/PMC3427763/pdf/nihms277146.pdf.

3. Azevedo DM, Azevedo IC, Holanda CSM, Santos QG, Vale LD, Cassiano AN. Da academia à realidade: uma reflexão acerca da prática do exame físico nos serviços de saúde. Sal \& Transf Soc. 2013 [Citado em 2019 Out. 28]; 4(4):106-110. Disponível em: http://incubadora.periodicos.ufsc.br/index. php/saudeetransformacao/article/view/1550

4. Cunha SMB, Barros ALBL. Análise da implementação da sistematização da assistência de enfermagem, segundo o modelo conceitual de Horta. Rev Bras Enferm. 2005 [Citado em 2019 Out. 28]; 58(5): 568-72. Disponível em: http://www.scielo.br/scielo.php?pid=S003471672005000500013\&script=sci_abstract\&tlng=pt.

5. Santos N, Veiga P, Andrade R. Importância da anamnese e do exame físico para o cuidado do enfermeiro. Rev. Bras. Enferm. 2011 [Citado em 2019

Out. 28]; 64(2):355-58. Disponível em: http://www.scielo.br/scielo.php?script=sci_arttext\&pid=S0034-71672011000200021

6. Kletemberg DF, Siqueira MD, Mantovani MF. Uma história do processo de enfermagem nas publicações da Revista Brasileira de Enfermagem no período 1960-1986. Esc Anna Nery R Enferm 2006 dez [Citado em 2020 Jan. 09]; 10 (3): 478 - 86. Disponível em: http://www.scielo.br/pdf/ean/v10n3/ v10n3a17.pdf

7. Silva CMC, Valente GSC, Saboia VM, Teixeira ER. 0 exame físico e o processo de enfermagem: para além do dualismo entre teoria e prática. Rev. Enferm UFPE. 2014 [Citado em 2019 Out. 28]; 8(supl.1):2281-6. Disponível em: https://periodicos.ufpe.br/revistas/revistaenfermagem/article/download/9916/10194

8. BRASIL. Conselho Federal de Enfermagem. Resolução $n^{\circ} 358$ de 15/10/2009. Dispõe sobre a sistematização da assistência de enfermagem e a implementação do processo de enfermagem em ambientes públicos e privados em que ocorre o cuidado profissional de enfermagem e dá outras providências. Diário Oficial da União. Brasília, 23 out. 2009, Seção 1, p.179. [Citado em 2019 Out. 28]. Disponível em: http://www.cofen.gov.br/resoluo-cofen-3582009 4384.html

9. Adamy EK, Mendes M, Schimitt MD, Maia JC, Brum MLB, Vendruscolo C. Formação de enfermeiros sobre anamnese e exame físico. J Nurs Health. 2016; [Citado em 2019 Dez. 16]; 6(2): 334-45. Disponivel em: https:// periodicos.ufpel.edu.br/ojs2/index.php/enfermagem/article/view/6767/6050 10. Sousa VD, Barros ALBL. 0 ensino do exame físico em escolas de graduação em enfermagem do município de São Paulo. Rev Lat Am Enfermagem 1998 [Citado em 2019 Out. 28]; 6(3):11-22. Disponível em: http://www.scielo.br/pdf/rlae/v6n3/13887.pdf

11. Birks M, Cant R, James A, Chung C, Davis J. The use of physical assessment skills by registered nurses in Australia: Issues for nursing education. Collegian. 2013 [Citado em 2019 Out. 28]; 20(1):27-33. Disponível em: https://www.ncbi.nlm.nih.gov/pubmed/23678781

12. Xavier LF, Silva SBM, Silva YCO, Oliveira OD, Morais Júnior SLA. Sistematização da assistência de enfermagem: o conhecimento de enfermeiros do município de JI-Paraná, Rondônia, Brasil. Revista Nursing. 2018 [Citado em 2020 Jan. 09]; 21 (239): 2110-2113. Disponivel em: http://www.revistanursing.com.br/revistas/239-Abril2018/sistematizacao assistencia_de_enfermagem.pdf

13. Carvalho EC, Oliveira-Kumakura ARS, Morais SCRV. Clinical reasoning in nursing: teaching strategies and assessment tools. Rev Bras Enferm. 2017[Citado em 2020 Mar. 09];70(3):662-668. doi: 10.1590/0034-71672016-0509. Disponível em: http://www.scielo.br/pdf/reben/v70n3/pt 0034-7167-reben-70-03-0662.pdf

14. Riegel $F$, Junior NJO. Processo de enfermagem: implicações para a segurança do paciente em centro cirúrgico. Cogitare Enferm. 2017 [Citado em 2020 Mar. 09]; 22(4): 01-05. doi: 10.5380/ce.v22i1.45577. Disponível em: http://docs.bvsalud.org/biblioref/2017/10/859852/45577-194188-1-pb.pdf 15. Trindade LR, Ferreira AM, Silveira A, Rocha EM. Processo de enfermagem: desafios e estratégias para sua implementação sob a ótica de enfermeiros. Saúde (Santa Maria) 2016 [Citado em 2020 Mar. 09];42(1):75-82. Doi: http://dx.doi.org/10.5902/2236583419805 Disponivel em: https://periodicos.ufsm.br/revistasaude/article/view/19805

16. Delaney MM, Friedman MI, Fitzpatrick JJ. Impacto of a Sepsis Educational Program on Nurse Competence. The Journal of Continuing Education in Nursing. 2015 [Citado em 2019 Dez. 16]; 46(4):179-186. Disponível em: https://www.healio.com/nursing/journals/jcen/2015-4-46-4/\%7B682b52b2-aaae-4d4c-ac55-96019053e58e\%7D/impact-of-a-sepsis-educational-program-on-nurse-competence

17. Storozuk AS, MacLeod MLP, Freeman S, Banner D. A survey of sepsis knowledge among Canadian emergency department registered nurses. Australian Emergency Care. 2019 Jun [Citado em 2019 Dez. 16]; 22(2):119125. Disponivel em: https://www.sciencedirect.com/science/article/abs/pii/ S2588994X19300077?via\%3Dihub

18. Douglas C, Windsor C, Lewis P. Too much knowledge for a nurse? Use of physical assessment by final-semester nursing students. Nurs Health Sci. 2015 [Citado em 2019 Dez. 16]; 17(4):492-9. Disponível em: https://onlinelibrary.wiley.com/doi/abs/10.1111/nhs.12223

19. Giddens JF. A Survey of Physical Assessment Techniques Performed by RNs: Lessons for Nursing Education. J Nurs Educ. 2007 [Citado em 2019 Out. 28]; 6(2):83-7. Disponível em: https://www.ncbi.nlm.nih.gov/pubmed/17315568

20. Gharaibeh B, Al-Smadi AM, Ashour A, Slater P. Development and psychometric testing of the Physical Examination Attitudes and Practices Scale. Nurs Forum. 2019[Citado em 2020 Mar. 09];54(1):111-120. doi: 10.1111/ nuf.12304. Disponível em: https://onlinelibrary.wiley.com/doi/full/10.1111/ nuf.12304

21. Silber JH, Rosenbaum PR, McHugh MD, Ludwig JM, Smith HL, Niknam BA, Even-Shoshan 0, Fleisher LA, Kelz RR, Aiken LH. Comparison of the Value of Nursing Work Environments in Hospitals Across Different Levels of Patient Risk. JAMA Surg. 2016 [Citado em 2020 Mar. 09];151(6):527-36. doi: 10.1001/jamasurg.2015.4908. Disponível em: https://www.ncbi.nlm.nih. gov/pmc/articles/PMC4957817/

22. Birks M, James A, Chung C, Cant R, Davis J. The teaching of physical assessment skills in pre-registration nursing programmes in Australia: issues for nursing education. Collegian. 2014 [Citado em 2019 Out. 28]; 21(3):24553. Disponivel em: https://www.ncbi.nlm.nih.gov/pubmed/25632720 